\title{
Granuloma piógeno oral recurrente con pérdida ósea alveolar y movilidad dentaria: Reporte de un caso inusual
}

\author{
Recurrent oral pyogenic granuloma with alveolar bone \\ loss and dental mobility: Report of an unusual case \\ Granuloma piogênico oral recorrente com perda óssea alveolar \\ e mobilidade dental: Relato de um caso incomum
}

\author{
Sandro Alexander Lévano Loayza' (iD) 0000-0002-2603-989X \\ Alfredo Yupanqui Pellanne ${ }^{2}$ (D) 0000-0002-7735-4013
}

\section{Resumen}

El granuloma piógeno oral es una lesión benigna multifactorial, caracterizada por presentarse como un agrandamiento gingival muy vascularizado. Se puede localizar en cualquier área de la cavidad oral, con más frecuencia en la encía marginal vestibular. Se presenta con mayor incidencia en mujeres adultas y en niños varones. No suele comprometer tejido óseo ni dientes y su tratamiento más seguro es la exéresis quirúrgica, siendo el riesgo de recurrencia alto. El objetivo del presente estudio es reportar el caso de una paciente de 9 años de edad, que fue sometida a la exéresis de un granuloma piogénico oral en el hueso maxilar y al año siguiente presentó una recurrencia de la lesión con pérdida ósea alveolar y movilidad de un diente adyacente. Se le realizó una biopsia y un curetaje minucioso, confirmándose el diagnostico de granuloma piogénico oral.

Palabras clave: granuloma piogénico, granuloma de células gigantes, diagnóstico.

Facultad de Estomatología, Universidad Peruana Cavetano Heredia, Perú. sandro.levano.1@gmail.com

Departamento Académico de Medicina y Cirugía Bucomaxilofacial, Facultad de Estomatología, Universidad Peruana Cayetano Heredia, Perú.

Fecha de recibido: 30/6/2020 - Fecha de aceptado: 8/11/2020 


\begin{abstract}
Oral pyogenic granuloma is a benign multifactorial lesion, characterized by having a gingival enlargement highly vascularized. It can be located in any area of the oral cavity, most often in the vestibular marginal gingiva. It occurs with a higher incidence in adult women and in male children. It does not usually compromise bone tissue or dental structures and its safest treatment is surgical excision, being the risk of recurrence high. The objective of the present study is to report the case of a 9-year-old female patient, who underwent excision of an oral pyogenic granuloma in the maxilla and the following year presented a possible recurrence of the lesion with alveolar bone loss and the mobility of one adjacent tooth. A biopsy and a thorough curettage were performed, confirming the diagnosis of recurrent oral pyogenic granuloma.
\end{abstract}

Keywords: pyogenic granuloma, giant cell granuloma, diagnosis.

\section{Introducción}

El granuloma piógeno es una proliferación vascular neoplásica benigna que se desarrolla en la piel y la cavidad oral. Erróneamente denominado "piógeno" debido a que no es un proceso infeccioso y no produce secreción purulenta. También se lo ha denominado granuloma pediculado benigno, hemangioma capilar lobular, épulis vascular o granuloma gravídico y tumor del embarazo cuando se presenta en pacientes gestantes $^{(1-3)}$.

\section{Resumo}

O granuloma piogênico oral é uma lesão multifatorial benigna, caracterizada por apresentarse como um aumento gengival altamente vascularizado. Pode estar localizado em qualquer área da cavidade oral, mais frequentemente na gengiva marginal vestibular. Ocorre com maior incidência em mulheres adultas e em crianças do sexo masculino. Geralmente não compromete o tecido ósseo ou os dentes e seu tratamento mais seguro é a escisão cirúrgica, sendo alto o risco de recorrência. O objetivo do presente estudo é relatar o caso de uma paciente de 9 anos de idade, submetida a escisão de granuloma piogênico oral no maxilar e no ano seguinte apresentou uma recorrência da lesão com perda óssea alveolar e a mobilidade de umo de seus dentes adjacentes. Uma biópsia e uma curetagem completa foram realizadas, confirmando o diagnóstico de granuloma piogênico oral.

Palavras-chave: granuloma piogênico, granuloma de células gigantes, diagnóstico.

\section{Antecedentes}

El granuloma piógeno se puede desarrollar en cualquier parte de la región de la cabeza y el cuello, tronco y extremidades, y cuando se presenta en la cavidad oral se le denomina Granuloma Piógeno Oral (GPO) teniendo como principal lugar de aparición la zona gingival, pero también se puede presentar en la mucosa bucal, carrillos, paladar, labios y lengua en menor frecuencia ${ }^{(4-6)}$. Su etiología aun es desconocida, pero se cree que está asociado a traumatismo crónico, irritantes locales, irritación por cálculo dental producto de una mala higiene bucal, medicamentos como la ciclosporina y cambios hormonales ${ }^{(7,8)}$. 
Clínicamente se caracteriza por ser una lesión de color rojizo, que puede mostrar ulceraciones y de fácil sangrado, de superficie suave y lobulada, con base sésil o pediculada ${ }^{(9)}$. Usualmente es una lesión de tamaño pequeño $(<2 \mathrm{~cm})$ y por lo general no causa ningún cambio en el hueso alveolar ${ }^{(10)}$. No tiene predilección en cuanto a edad y sexo, pero es más frecuente en la segunda década de vida y en el sexo femenino ${ }^{(11-13)}$. Histológicamente se le puede clasificar en hemangioma capilar lobular y hemangioma capilar no lobular. No obstante, el primero presenta además de un revestimiento endotelial una capa de músculo liso, la cual es característica de las vénulas y no de los capilares, por lo que el nombre más apropiado para esta entidad sería el de hemangioma lobulado ${ }^{(14)}$.

Debido a sus características posee diversidad de diagnósticos diferenciales como fibroma odontogénico periférico, hemangioma, hiperplasia gingival inflamatoria, granuloma periférico de células gigantes, linfoma no Hodgkin, angiosarcoma, fibroma osificante periférico, sarcoma de Kaposi entre otros ${ }^{(3,5)}$.

El tratamiento de elección es la exéresis quirúrgica, algunos autores reportan no tener recurrencia con este método ${ }^{(15,16)}$. Sin embargo, la recurrencia está entre el 5.8\% y $16 \% \%^{(1,17)}$. Por otro lado, se ha evidenciado en investigaciones de seguimiento después de un año que no existe recurrencia de la lesión cuando se realiza una exéresis modificada con curetaje profundo ${ }^{(18)}$.

El objetivo del presente estudio es reportar el caso de una paciente de 9 años de edad, que fue sometida a la exéresis de un granuloma piógeno oral en el maxilar y al año siguiente presentó recurrencia de la lesión con pérdida ósea alveolar y movilidad dentaria de uno de sus dientes adyacentes. Se le realizó biopsia excisional y curetaje minucioso, confirmando el diagnostico de granuloma piógeno oral.

\section{Caso clínico}

Paciente de sexo femenino de 9 años de edad sin antecedentes sistémicos que acudió al Servicio de Cirugía Oral y Maxilofacial del Centro Dental Docente de la Universidad Peruana Cayetano Heredia por presentar aparentemente una recurrencia de una lesión en la región posterior del maxilar. La paciente relato que hace un año consultó por dicha lesión y que fue sometida a biopsia excisional, cuyo resultado anatomopatológico fue de granuloma piógeno, se le realizaron controles clínicos hasta darle de alta.

$\mathrm{Al}$ examen clínico intraoral de la consulta actual se evidenció una lesión de apariencia tumoral dolorosa a la palpación y a la masticación a nivel de encía marginal y alveolar de los dientes 1.4 y 1.5 (Fig. 1), de aproximadamente $3 \times 3 \times 3$ $\mathrm{cm}$ de diámetro, que cubría las caras vestibular, palatina y parte de la corona clínica de dichos dientes. Su consistencia era firme, de aspecto lobulado y pediculado, y textura rugosa y sangrante ante el estímulo, con movilidad grado II del diente 1.4 (Fig. 2). Al examen radiográfico se observó borramiento de la cresta alveolar entre dientes 1.5 y 1.4 con mesioangulación y ensanchamiento del espacio periodontal del diente 1.4 (Fig. 3). En base a la evolución y características clínicas se planteó el diagnóstico presuntivo de granuloma piógeno o granuloma periférico de células gigantes.

Fig. 1: Vista frontal de boca en oclusión evidenciando lesión en cuadrante I

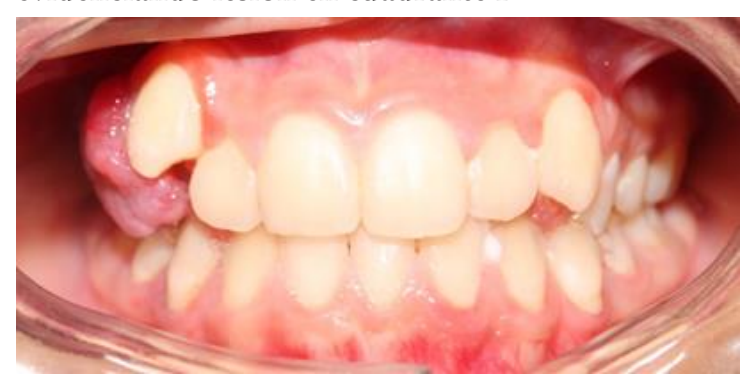


Fig. 2. A: Vista frontal de la lesión cubriendo las caras vestibulares de los dientes 1.4 y 1.5. B: Vista oclusal de la lesión cubriendo las caras palatinas y parte de la corona clínica de los dientes 1.4 y 1.5
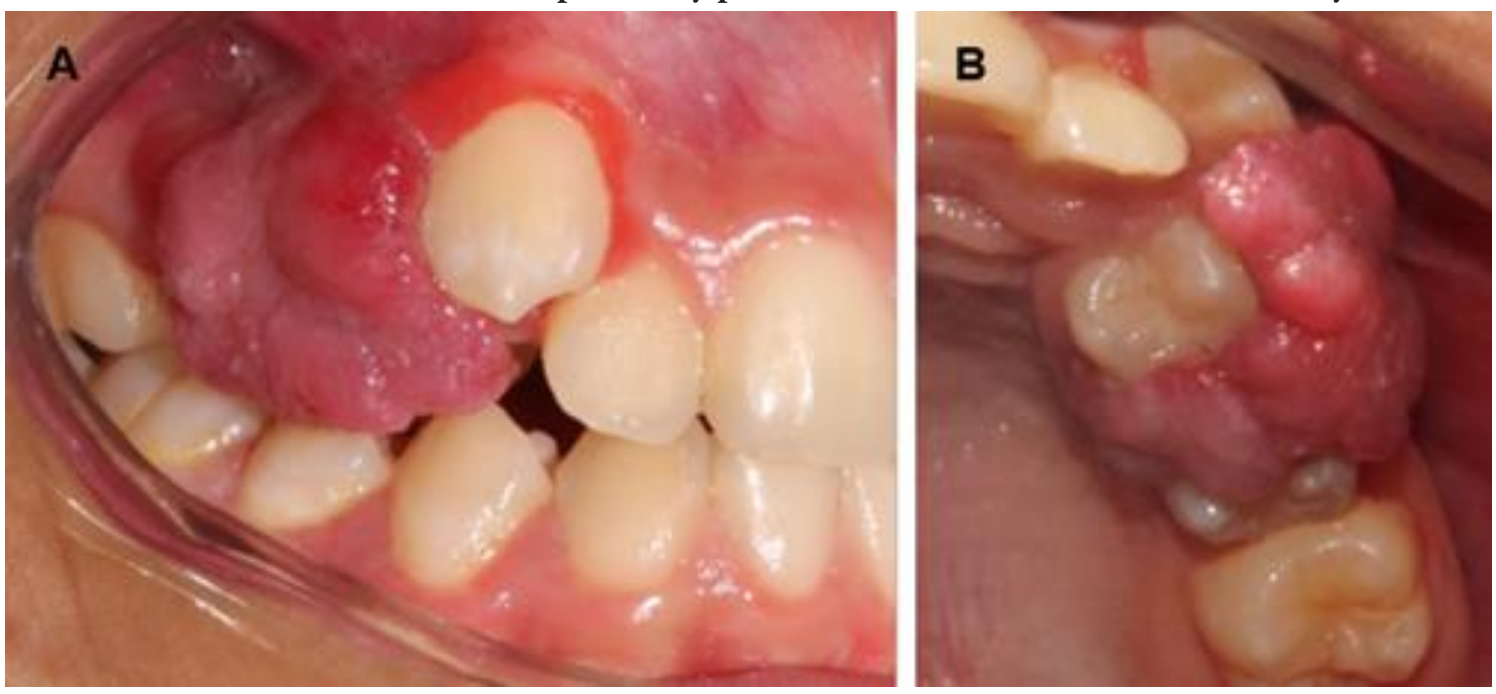

Fig. 3: Radiografía periapical de la región afectada por la lesión que muestra una clara pérdida ósea alveolar en sentido horizontal entre los dientes 1.4 y 1.5. Además, se evidencia mesioangulación del diente 1.4

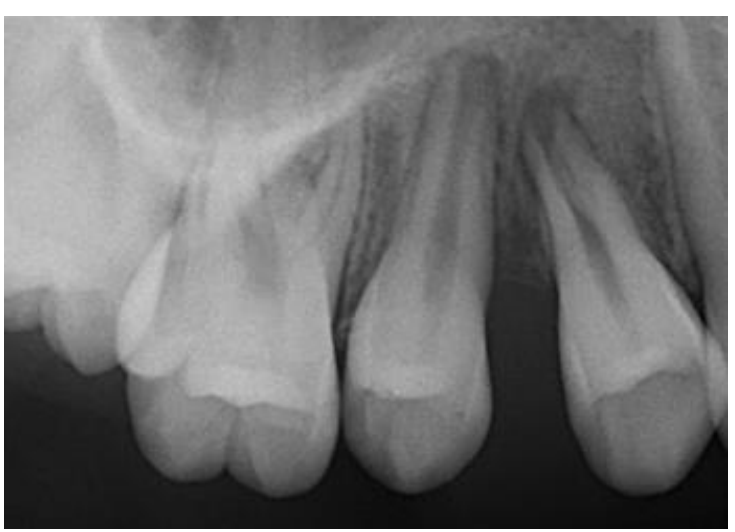

Se procedió a realizar un colgajo rotacional, una biopsia excisional y un curetaje minucioso de tejido sano removiendo el periostio adyacente entre 2 a $3 \mathrm{~mm}$ de extensión y posteriormente se suturó.

Se realizaron controles clínicos a los 7 días (Fig. 4) y posteriormente a la semana. La herida quirúrgica no presentó dehiscencias ni secreciones en proceso de cicatrización favorable con puntos de sutura estables. El resultado anatomopatológico fue granuloma piógeno oral, lo que confirmó su recurrencia.
Fig. 4: Control clínico después de 7 días de evolución, no presentando dehiscencias ni secreciones y en un proceso de cicatrización normal y favorable.

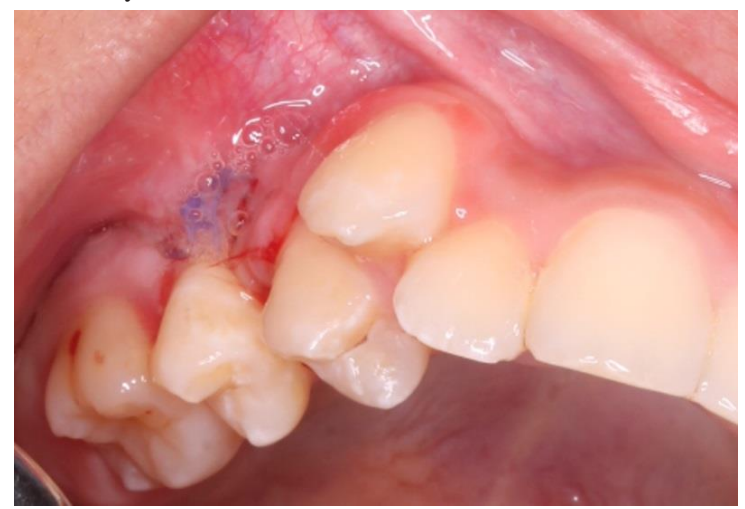

\section{Discusión}

El diámetro del GPO recurrente $(3 \mathrm{~cm})$ fue mayor a lo publicado en la literatura. Sin embargo, Rebolledo et al., ${ }^{(19)}$ han informado que puede llegar a medir hasta $4.5 \mathrm{~cm}$ de diámetro.

Según la edad, el granuloma piógeno oral es más frecuente en mujeres adultas que en varones. No obstante, en niños varones se desarrollan GPO más frecuentemente que en las niñas ${ }^{(20)}$, por lo que el presente caso no se ajusta a las características epidemiológicas frecuentes por edad y sexo. 
La reabsorción radicular causada por la presencia del granuloma piógeno oral es una condición rara, que generalmente ocurre en casos de larga evolución ${ }^{(21)}$. Una pérdida extensa de hueso alveolar y la movilidad dentaria se observa en el 3\% de los casos ${ }^{(22)}$. Shenoy et al., ${ }^{(23)}$ reportaron el caso de un niño de 8 años de edad con granuloma piógeno oral asociado a reabsorción ósea y Goodman-Topper et al., ${ }^{(24)}$ también reportaron otro caso de granuloma piógeno oral con pérdida ósea alveolar en un niño de 12 años de edad. Además, Ababneb et al., ${ }^{(25)}$ reportaron el caso de una gestante de 28 años de edad con la misma lesión y característica de pérdida ósea alveolar. En el presente caso observamos pérdida ósea alveolar en la lesión recurrente, característica poco frecuente del GPO. Es probable que al recurrir haya producido dicha reabsorción.

El diagnóstico diferencial de los GPO incluye fibroma odontogénico periférico, hemangioma, hiperplasia gingival inflamatoria, granuloma periférico de células gigantes, linfoma no Hodgkin, angiosarcoma, fibroma osificante periférico, sarcoma de Kaposi, linfangioma, sífilis, úlcera tuberculosa y úlcera traumática ${ }^{(3,5,26)}$. En nuestro caso, el GPO simulaba clínicamente la mayoría de las entidades anteriormente mencionadas como diagnósticos diferenciales y sumado a la característica de pérdida ósea y movilidad dentaria representó un desafío diagnóstico, por lo que fue de vital importancia el análisis anatomopatológico para un diagnóstico y tratamiento certero.

La recurrencia aparece en intervalos de 10 meses a 1 año ${ }^{(18)}$, periodo que coincide con el pre- sente caso. Se sabe además que la recurrencia estaría asociada a múltiples factores, siendo la más resaltante la mala higiene bucal en vez de un compromiso sistémico. En nuestro caso la paciente presentaba una higiene bucal regular por lo que, en este caso, aquel factor no fue determinante. No obstante, se le hizo énfasis en la mejoría de su higiene bucal para prevenir también otras enfermedades bucales. La extracción de $2 \mathrm{~mm}$ del tejido normal y el curetaje profundo ayudan a prevenir la recurrencia de la lesión ${ }^{(18)}$. Por ello realizamos un curetaje minucioso removiendo el periostio adyacente entre 2 a 3 mm de extensión.

\section{Conclusiones}

El granuloma piógeno oral es una lesión benigna bien conocida. Sin embargo, cuando no comparte similitud en sus características más comunes, como el tamaño promedio, evolución y características radiográficas, constituye un desafío en el diagnóstico, debido a que debe descartarse una lesión maligna.

El diagnóstico y tratamiento seguros del granuloma piógeno oral son la biopsia excisional y el curetaje minucioso, eliminando el periostio adyacente entre 2 a $3 \mathrm{~mm}$ de extensión, previniendo posibles recurrencias.

La recurrencia del granuloma piógeno oral puede dar lugar a la pérdida ósea alveolar con movilidad dentaria. Por lo tanto, el clínico debe realizar un tratamiento adecuado temprano para impedir el avance de la lesión.

\section{Referencias}

1. Al-Khateeb T, Abhabneh K. Oral pyogenic granuloma in Jordanians: a retrospective analysis of 108 cases. J Oral Maxillofac Surg. 2003; 61: 1285-8.

2. García I, Hinojosa A, Aldape B, Valenzuela E. Hemangioma lobular capilar (granuloma piógeno) asociado a la erupción: reporte de dos casos clínicos. Rev Odont Mex. 2004; 8(4): 127- 32.

3. Sharma S, Chandra S, Guptya S, Srivastava S. Heterogeneous conceptualization of etiopathogenesis. Oral pyogenic granuloma. Natl J Maxillofac Surg. 2019; 10: 3-7. 
4. Concepción L, Belmonte R, Acosta M, Torres E, Infante P, Torres D, Gutierrez JL. Manejo del granuloma piógeno gigante. Revista SECIB. 2005; 4: 74-89.

5. Kamal R, Dahiya P, Puri A. Oral pyogenic granuloma: Various concepts of etiopathogenesis. J Oral Maxillofac Pathol. 2012; 16(1): 79.

6. Papageorge MB, Doku HC. An exaggerated response of intraoral pyogenic granuloma during puberty. J Clin Pediatr Dent. 1992; 16(3): 213-6

7. Chandrashekar B. Minimally invasive approach to eliminate pyogenic granuloma: A case report. Case Reports in Dentistry. 2012; 26. doi: 10.1155/2012/909780

8. Isola G, Matarese G, Cervino G, Matarese M, Ramaglia L, Cicciù M. Clinical Efficacy and Patient Perceptions of Pyogenic Granuloma Excision Using Diode Laser Versus Conventional Surgical Techniques. J Craniofac Surg. 2018; 29(8): 2160-3.

9. Epivatianos A, Antoniades D, Zaraboukas T, Zairi E, Poulopoulos A, Kiziridou A, Iordanidis S. Pyogenic granuloma of the oral cavity: comparative study of its clinicopathological and immunohistochemical features. Pathol Int. 2005; 55: 391-7.

10. Thada SR, Pai KM, Agarwal P. A huge oral pyogenic granuloma with extensive alveolar bone loss and 'sun-ray' appearance mimicking a malignant tumour. BMJ Case Rep. 2014; 3: 101-36.

11. Dojcinovic I, Richter M, Lombardi T. Occurrence of a pyogenic granuloma in relation to a dental implant. J Oral Maxillofac Surg. 2010; 68 (8): 1874-6.

12. Silk H, Douglass AB, Douglass JM, Silk L. Oral health during pregnancy. Am Fam Physician. 2008; 77 (8): 1139-44.

13. Saravana GH. Oral pyogenic granuloma: a review of 137 cases. Br J Oral Maxillofac Surg. 2009; 47(4): 318-9.

14. Santa Cruz D, Plaza JA, Wick MR, Gru AA. Inflammatory lobular hemangioma (ILH): a vascular proliferation with a prominent lymphoid component. Review of a series of 19 cases. J Cutan Pathol 2020. Doi: $10.1111 /$ cup.13844.

15. Kaya A, Kaya B. Oral pyogenic granuloma. Review of 10 cases. Indian J Med Res Pharm Sci. 2015; 2: 44-8.

16. Tiwari S, Neelakanti A, Sathyanarayana S. An innovative and less invasive management of recurrent pyogenic granuloma in the esthetic zone: A case report with 18-month follow-up. J Indian Soc Periodontol. 2017; 21: 241-4.

17. Hasanoglu GN, Senguven B, Gultekin SE, Cetiner S. Management of a recurrent pyogenic granuloma of the hard palate with diode laser: A case report. J Lasers Med Sci. 2016; 7: 56-61.

18. Al-Noaman AS. Pyogenic granuloma: Clinicopathological and treatment scenario. J Indian Soc Periodontol. 2020; 24: 233-6.

19. Rebolledo M, Harris J, Cantillo O, Carbonell Z, Díaz A. Granuloma telangiectásico en cavidad oral. Av. Odontoestomatol. 2010; 26 (5): 249-53.

20. Mohapatra S, Singh K, Singh L, Kumar P. Oral pyogenic granuloma: A review. Journal of Odisha Dental Association. 2014; 3(1): 5-9.

21. Angelopoulos AP. Pyogenic granuloma of the oral cavity: Statistical analysis of its clinical features. J Oral Surg. 1971; 29: 840-7.

22. Silverstein L, Burton CH. Jr, Singh B. Oral pyogenic granuloma in pregnancy. Int. J. Gynaecol. Obstet. 1995 ; 49 : 331-2.

23. Shenoy SS, Dinkar AD. Pyogenic granuloma associated with bone loss in an eight-year-old child: a case report. J. Indian Soc. Pedod. Prev. Dent. 2006; 24: 201-3.

24. Goodman-Topper ED, Bimstein E. Pyogenic granuloma as a cause of bone loss in a twelve-year-old child: report of a case. ASDC J. Dent. Child. 1994; 61: 65-7.

25. Ababneb K, Al-Khateeb T. Aggressive pregnancy tumor mimicking a malignant neoplasm: a case report. J. Contemp. Dent. Pract. 2009; 10: E072-8, 2.

26. Singh RK, Kaushal A, Kumar R, Pandey RK. Profusely bleeding oral pyogenic granuloma in a teenage girl. BMJ Case Rep. 2013. Doi: 10.1136/bcr-2013-008583. 


\section{Nota de conflicto de interés:}

Los autores declaran no tener conflicto de interés en el artículo.

\section{Nota contribución de los autores:}

1. Concepción y diseño del estudio

2. Adquisición de datos

3. Análisis de datos

4. Discusión de los resultados

5. Redacción del manuscrito

6. Aprobación de la versión final del manuscrito

S.A.L.L ha contribuido en: 1, 2, 3, 4, 5 y 6 .

A.Y.P ha contribuido en: 1, 3, 4, 5 y 6 .

\section{Nota de aceptación:}

Este artículo fue aprobado por la editora de la revista Mag. Dra. Vanesa Pereira-Prado. 